\title{
Steroid Hormone Regulation of Ribosomal RNA in Rat Hypothalamus: Early Detection Using in situ Hybridization and Precursor-Product Ribosomal DNA Probes
}

\author{
Kathryn J. Jones, ${ }^{1,3}$ Christina A. Harrington, ${ }^{2,4}$ Dona M. Chikaraishi, ${ }^{2}$ and Donald W. Pfaff ${ }^{3}$ \\ 'Department of Cell Biology and Anatomy, The Chicago Medical School, North Chicago, Illinois 60064, ${ }^{2}$ Graduate Program \\ in Neuroscience, Tufts University School of Medicine, Boston, Massachusetts 02111, 3Laboratory of Neurobiology and \\ Behavior, The Rockefeller University, New York, New York 10021, and ${ }^{4}$ Cambridge NeuroScience Research, Inc., \\ Cambridge, Massachusetts 02139
}

In the female rat, behavioral and endocrine aspects of reproduction are controlled, in part, by the action of the steroid hormone estradiol on several regions of the brain, including the ventrolateral portion of the ventromedial hypothalamus (VL-VMN) and the arcuate nucleus of the hypothalamus (ARC). Quantitative assessment of the effects of estradiol on the regulation of ribosomal RNA in rat hypothalamus was accomplished in this study by tandem in situ hybridization experiments with 2 ribosomal DNA probes specific to the initial transcript (precursor) or mature, stable (product) rRNA. This novel approach allowed the regulation of RNA processing by steroid hormones to be analyzed in the individual neuron, a particularly important concern in heterogeneous tissue such as the brain. Estradiol was administered subcutaneously to ovariectomized rats for $15 \mathrm{~min}, 30 \mathrm{~min}$, or 2 $\mathrm{hr}$, or a discontinuous schedule of $2 \mathrm{hr}$ on $/ 7 \mathrm{hr}$ off/2 hr on. Levels of precursor and product rRNA were measured in VLVMN and ARC neurons using a computerized image-analysis system. Significant increases in the levels of precursor rRNA were observed only in the VL-VMN as early as $\mathbf{3 0}$ min after hormone exposure, with a doubling in the amount of precursor rRNA occurring at 2 hr. No changes in product rRNA were observed in either brain region at these early times. These data, in conjunction with our previous findings of increases in product rRNA after longer hormone exposure times, lead us to conclude that rRNA gene transcription is activated in rat hypothalamic neurons within $\mathbf{3 0}$ min. This is the earliest effect of a steroid hormone on the neuronal genome reported to date and, as such, may represent a primary event in the activation of neuronal pathways involved in female reproductive behavior.

Ample evidence, accumulated from a number of neuronal systems, has firmly established that gonadal steroid hormones play a key role in brian function (Pfaff, 1980; Breedlove, 1986; DeVoogd, 1986). The mechanism by which steroids accomplish

\footnotetext{
Received May 1, 1989; revised Oct. 19, 1989; accepted Nov. 27, 1989

The technical assistance of Marie Helene Charlap and Theresa Cox is greatly appreciated. This study was supported in part by BRSG SO7RRO5366, DRR, NIH (K.J.J.) and NIH HDOS/SI (D.W.P.).

Correspondence should be addressed to Dr. Kathryn J. Jones, Department of Cell Biology and Anatomy, The Chicago Medical School, 3333 Green Bay Road, North Chicago, IL 60064.

Copyright (c) 1990 Society for Neuroscience $0270-6474 / 90 / 051513-09 \$ 02.00 / 0$
}

their effect on neurons is not as well-defined as in peripheral target tissues; however, given that many hormonally responsive brain regions contain steroid hormone receptors (Morrell and Pfaff, 1983), it is likely that steroids act neuronally through classically described receptor-mediated gene activation (Jensen et al., 1982). From a functional perspective, the role of estradiol in regulating the behavioral and neuroendocrine components of reproduction in the female rat has been substantially characterized (Pfaff, 1980). Estradiol implant (Barfield and Chen, 1977; Davis et al., 1982), lesion (Mathews and Edwards, 1977), steroid hormone autoradiographic (Pfaff and Keiner, 1983), and electrophysiological (Moss and Foreman, 1976) studies have shown that the anatomical substrate mediating the effects of estradiol on reproductive behavior lies in the neurons of the ventrolateral portion of the ventromedial nucleus of the hypothalamus (VLVMN). It is in this hormonally responsive neuronal system that significant progress has been made in elucidating the mechanism of steroid hormone action on nerve cells (Jones, 1988).

Initial progress in determining the effects of estradiol on individual VL-VMN neurons was accomplished through the ultrastructural studies of Cohen and Pfaff (1981). These investigators found significant alterations in protein secretory components of VMN neurons following long-term exposure of ovariectomized (OVX) rats to estradiol. Since it has been shown in non-neural target tissue that steroids effect changes in cellular activity within hours, we extended the studies of Cohen and Pfaff by cxamining the cffects of short cstradiol cxposure times on VL-VMN neurons. Morphological signs of widespread genomic activation were found after just $2 \mathrm{hr}$ of a physiologically relevant dose of estradiol (Jones et al., 1985). These changes included alterations in the nucleolus, rough endoplasmic reticulum (RER), and the DNA-RNA network within the nucleoplasm, increases in cell and nuclear size, and transformation in nuclear shape toward spherical. A second 2 hr pulse of estradiol, $7 \mathrm{hr}$ after the first $2 \mathrm{hr}$ pulse of the hormone, resulted in progressive changes in the RER and somal and nuclear swelling, but a regression toward the control of the nuclear shape changes and nucleolar size. These data, as well as the results of protein synthesis studies in the same system and/or under the same time points of hormone treatment (Scouten et al., 1985; Jones et al., 1987, 1988; Mobbs et al., 1988), have led to the hypothesis that exposure to estradiol results in a cascade of events within VL-VMN neurons that includes rapid changes in genomic activity and differential expression of proteins at early and later 
time points. From parallel studies with other estradiol-concentrating neurons, several tenets regarding hormone action in the brain have begun to emerge. First, the actions of steroid hormones appear to exhibit brain region specificity, in terms of both structural and chemical effects (Meisel and Pfaff, 1985; Jones et al., 1986, 1987, 1988). Second, the action of estradiol on VMN neurons is the most pronounced of any estradiolconcentrating neurons examined to date, and at some stages appears to be very similar to tissue within the reproductive tract (Vic et al., 1980).

Given both the importance of the polymerase I system in protein secretory cells such as neurons and the correlation between nucleolar and protein synthetic activities (Busch and Smetana, 1970; Hadjiolov, 1985), we recently initiated a series of in situ hybridization experiments using stable ribosomal DNA (rDNA) probes to measure estradiol-induced changes in gene expression within individual VL-VMN neurons (Jones et al., 1986). Within $6 \mathrm{hr}$ of exposure of OVX rats to estradiol, the minimal continuous hormone replacement paradigm necesary to activate progesterone-facilitated lordosis behavior, a $70 \%$ increase in levels of rRNA was found in VL-VMN neurons. From this discovery of a relatively early and robust effect of estradiol on cytosolic rRNA levels, we hypothesized that estradiol acts on hypothalamic neurons through a mechanism involving an increase in rRNA transcription. In this study, we tested that hypopthesis with in situ hybridization experiments in which both precursor rDNA and product (stable) rDNA levels were measured in individual VL-VMN and ARC neurons after very short exposure of OVX rats to estradiol or a behaviorally relevant discontinuous schedule of hormone exposure consisting of two $2 \mathrm{hr}$ pulses of estradiol separated by $7 \mathrm{hr}$. The discontinuous paradigm of hormone administration was described by Parsons et al. (1982a, b), who determined that it afforded adequate estradiol priming for progesterone-facilitated lordosis behavior at $24 \mathrm{hr}$. The use of rDNA probes encoding the external transcribed spacer region near the initiation site of $45 \mathrm{~S}$ precursor rRNA transcription as well as the region encoding the 28S rRNA subunit near the end of the ribosomal transcription unit provided a novel means of examining steroid hormone effects on the kinetics of ribosome formation in the individual neuron.

\section{Materials and Methods}

Animals and hormone administration. Female Long-Evans rats weighing 200-225 gm were ovariectomized (OVX) under methoxyfluranc anesthesia 5-7 d prior to the start of experimentation. Crystalline estradiol $\left(\mathrm{E}_{2}\right)$ was packed into $5 \mathrm{~mm}$ lengths of Silastic tubing and incubated in $0.1 \%$ PBS for $24 \mathrm{hr}$ prior to experimental use. Three to four OVX rats per group were anesthetized as described and subcutaneously implanted with $E_{2}$ capsules for $15 \mathrm{~min}, 30 \mathrm{~min}$, or $2 \mathrm{hr}$, or a discontinuous schedule of $2 \mathrm{hr}$ on, $7 \mathrm{hr}$ off, and $2 \mathrm{hr}$ on. OVX rats, anesthetized and sham-implanted under the same time course, served as controls.

Tissue preparation and fixation. At the end of the appropriate hormone exposure time, the animals were killed by decapitation. The brains were quickly removed, blocked to include the posterior portion of the mediobasal hypothalamus (anterior cut $=$ immediately posterior to the optic chiasm and posterior cut $=$ posterior to the median eminence; Pfaff, 1980), and fresh-frozen as described elsewhere (McCabe ct al., 1986). Every twelfth $3 \mu \mathrm{m}$ cryostat section throughout the region containing the ventromedial nucleus (VMN) and the arcuate nucleus (ARC) was collected on chrome-alum-subbed glass slides (see figures L-P in Pfaff and Keiner, 1973). Half of the slides, or approximately 5 sections, were used for hybridization with each of the probes. Thus, every 24 th $3 \mu \mathrm{m}$ (72 linear $\mu \mathrm{m}$ ) section was potentially available for quantification. Given that the average diameter of VMN and ARC neurons is approximately $15 \mu \mathrm{m}$ (Jones et al., 1985), this method of section collection eliminated the possibility of double counting. The sections were then fixed according to the protocol of McCabe et al. (1986) by immersion into $800 \mathrm{ml}$ volumes of the following solutions prepared under RNase-free conditions: $100 \%$ ethanol : acetic acid $(3: 1$ $\mathrm{vol} / \mathrm{vol}$ ) for $15 \mathrm{~min} ; 160 \mu \mathrm{l}$ fresh diethylpyrocarbonate in $800 \mathrm{ml}$ of $2 \times$ SSC titrated to $\mathrm{pH} 3.5$ with acetic acid $(2 \times$ SSC $=0.30 \mathrm{~m}$ sodium chloride, $0.03 \mathrm{~m}$ sodium citrate in autoclaved, doubly distilled water) for $30 \mathrm{~min}$ at $70^{\circ} \mathrm{C} ; 1 \mu \mathrm{g} / \mathrm{ml}$ pepsin in $2 \times$ SSC-acetic acid, $\mathrm{pH} \mathrm{3.5,} \mathrm{for}$ $15 \mathrm{~min}$ at $37^{\circ} \mathrm{C}, 0.2 \times \mathrm{SSC}$ for $1 \mathrm{~min}$ (twice): and $5 \mathrm{~mm}$ dithiothreitol in water. The sections were then dried overnight in a vacuum desiccator.

Ribosomal DNA probes. Nick-translated ribosomal DNA (rDNA) probes were prepared with ${ }^{3} \mathrm{H}-\mathrm{dCTP}(30 \mathrm{Ci} / \mathrm{mmol}, \mathrm{ICN})$ and ${ }^{3} \mathrm{H}-\mathrm{dTTP}$ $(60 \mathrm{Ci} / \mathrm{mmol}, \mathrm{ICN})$ as described by Lewis et al. (1983). The average denatured fragment length was around 30-50 bases, and the specific activity was approximately $10^{7} \mathrm{dpm} / \mu \mathrm{g}$. Plasmid pEE 6.7 contained 6.7 $\mathrm{kb}$ that included approximately $4 \mathrm{~kb}$ of mature $28 \mathrm{~S}, 5.8 \mathrm{~S}$, and $18 \mathrm{~S}$ rRNA coding region and $2.7 \mathrm{~kb}$ of internal transcribed spacer (Chikaraishi et al., 1983). Plasmid pHE 5.9 contained both the external transcribed spacer region (ETS) and the $18 \mathrm{~S}$ RNA coding region. From pHE 5.9, a $1.8 \mathrm{~kb}$ sequence of only ETS was excised with Xho I. This precursor fragment $(X X 1.8)$ was then separated by agarose gel electrophoresis, purified, and nick-translated.

In situ hybridization. The procedure of McCabe et al. (1986) was followed for the prehybridization, hybridization, and posthybridization steps. Included in this protocol are a number of specificity controls routinely incorporated into all experiments using the ribosomal DNA probes. The prehybridization (PHB) and hybridization $(\mathrm{HB})$ buffers contained the following reagents per $5 \mathrm{ml}$ autoclaved, doubly distilled water: $1.2 \mathrm{ml}$ of $5.0 \mathrm{M}$ sodium chloride; $100 \mu \mathrm{l}$ of $1.0 \mathrm{M}$ Tris $7.6 ; 16.7$ $\mu \mathrm{l}$ of $6 \%(\mathrm{wt} / \mathrm{vol}) \mathrm{BSA} ; 40 \mu \mathrm{l}$ of $250 \mathrm{~mm}$ EDTA; $50 \mu \mathrm{l}$ of $6 \%$ Ficoll 400 ; $33 \mu \mathrm{l}$ of $6 \%$ polyvinylpyrrolidine; $10 \mu \mathrm{l}$ of $50 \mathrm{mg} / \mathrm{ml}$ yeast tRNA; $25 \mu \mathrm{l}$ (HB) or $250 \mu \mathrm{l}(\mathrm{PHB})$ of $20 \mathrm{mg} / \mathrm{ml}$ yeast total RNA (Type $11 \mathrm{l}$ ); $100 \mu \mathrm{l}$ (HB) or $500 \mu \mathrm{l}$ (PHB) of $10 \mathrm{mg} / \mathrm{ml}$ herring sperm DNA (Type IV); and $5 \mu \mathrm{l}$ of $15 \mathrm{mg} / \mathrm{ml}$ polyoxyadenylic acid. PHB, $20 \mu \mathrm{l}$, diluted with formamide (1:1) was micropipetted onto each section and allowed to remain for $2 \mathrm{hr}$. Ribosomal DNA probes were dissolved in $\mathrm{HB}$, denatured by boiling, and then diluted by $50 \%$ with formamide. The HB containing the probe was prepared so that a radioactivity concentration of 2000 $\mathrm{cpm} / 20 \mu \mathrm{l} \mathrm{HB}$ :formamide (1:1) was achieved, and, after draining the PIIB from the slides, $20 \mu \mathrm{l}$ was micropipetted onto each section. The hybridization was done at room temperature for $18 \mathrm{hr}$. The slides were subsequently rinsed in $2 \times$ SSC twice for $10 \mathrm{~min}$ and washed overnight in 2 liters of $0.5 \times$ SSC containing $5 \mathrm{~mm}$ dithiothreitol. After final rinsing in successive solutions of ammonium acetate: ethanol, the slides were dried in a desiccator.

Autoradiography. Standard procedures for liquid emulsion autoradiography were followed. Briefly, under safelight conditions, randomly selected pairs of experimental and control slides were dipped in undiluted Kodak NTB-2 emulsion and allowed to dry in a light-tight box containing desiccant for $2 \mathrm{hr}$. The slides were then transferred to opaque slide boxes containing desiccant, sealed with black tape, and stored at $4^{\circ} \mathrm{C}$. At the appropriate exposure time (between 4-6 weeks), as determined by test slides, all slides were developed, lightly stained with cresyl violet, and coverslipped.

Quantitative procedures. As mentioned above, sections were collected in a systematic random sampling approach, which has been shown to yield smaller errors than random sampling (Cochran, 1953; Weibel, 1973). This approach was chosen because it avoids the problem of clustering, an important point given that only $30 \%$ of the VL-VMN and the ARC neurons are estradiol-concentrating (Morrell and Pfaff, 1983). To insure that a large percentage of the cells analyzed were estradiolconcentrating, sections were collected from the posterior portion $(0.3-$ $0.4 \mathrm{~mm}$ ) of the mediobasal hypothalamus, which contains the greatest density of estradiol-concentrating neurons. Thus, by systematic random sampling, we ensured that a representative portion of all the neurons within the posterior portion of both nuclear groups would be quantified. From approximately 5 sections per brain region of each animal and for cach probc, a minimum of 100 neurons was collected, resulting in standard errors $<10 \%$ of the mean (Bolender, 1970). Throughout the experimental procedures, paired experimental and control slides (e.g., 15 min $E_{2}$-treated and 15 min OVX, sham-implanted control) were processed together. Given the large number of slides per in situ experiment, it was not possible to process all slides across every hormone time point together. Thus, quantitative comparisons were made only between paired $E_{2}$-treated and OVX control animals, and not between the different 

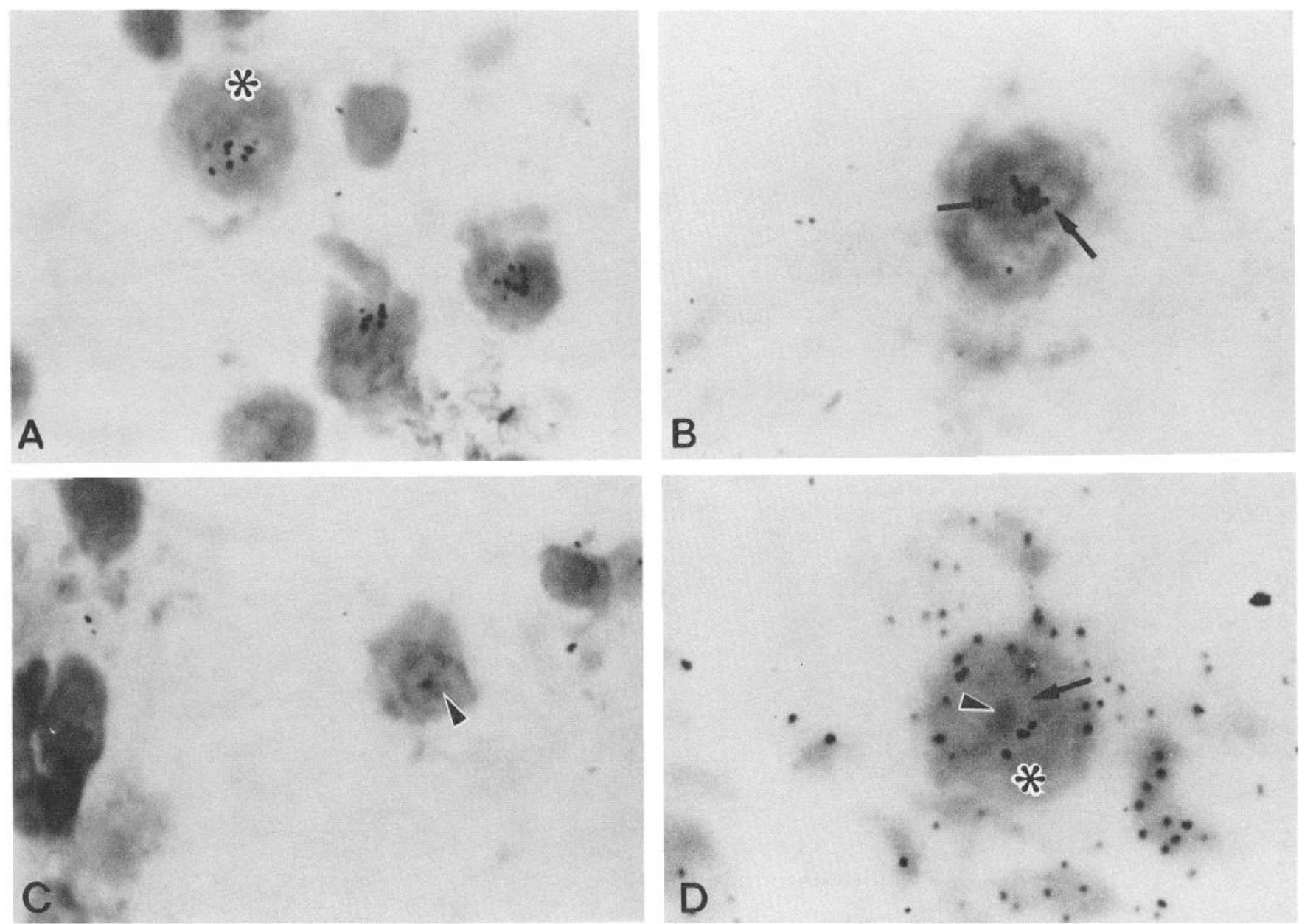

Figure 1. Autoradiographs of neurons from the ventrolateral portion of the ventromedial hypothalamus (VL-VMN) after hybridization with the precursor rDNA probe $(A-C)$ or product rDNA probe. In $A$, note the centrally located cluster of grains within the nucleus of several VL-VMN neurons, as well as the lack of labeling of the cytoplasm $\left(^{*}\right)$. In $B$, the nuclear outline can be seen (arrows), with a cluster of grains lying centrally within the confines of the nucleus. In $C$, a VL-VMN neuron from an OVX rat is illustrated. Light labeling allows visualization of the nucleolus (arrowhead), which, in a more heavily labeled cell, would be obscured by grains. In $D$, the pattern of grain distribution observed following hybridization with the product rDNA probe is characteristic of the location of mature ribosomes in VL-VMN neurons.

hormone treatment groups. Using a computerized image-analysis system (SMI-MicroComp, Atlanta, GA), the average number of grains per neuron, area of individual neurons, and grain density (number of grains/ unit area of soma) in $2 \mathrm{E}_{2}$-concentrating brains regions, VL-VMN (ventrolateral portion of the VMN) and the ARC, were measured. The area of an individual grain was measured in square microns, with the imageanalysis system, following photographic development of the emulsion. This measurement was kept constant for all quantitative procedures. Therefore, any clustering of grains was automatically divided by the area of a grain, with the result being a determination of the number of grains comprising a cluster. This computerized definition of a grain eliminated any possible bias on the part of the investigator. As an additional internal control, measurements were also collected from the dorsomedial portion of the VMN (DM-VMN), an area with few $\mathrm{E}_{2}$ concentrating neurons and a region that was contained in the same sections as the VL-VMN. Within each region, every neuron with a clearly defined somal contour was measured. All slides were coded by an uninvolved investigator in order to accomplish the analysis under "blind" conditions. While it is generally necessary to subtract background levels of labeling in order to correct for nonspecific hybridization, the background levels of labeling following in situ hybridization with both rDNA probes was essentially negligible. Statistical analysis of the data was accomplished using 2-way ANOVA ( 2 factors $=$ hormone treatment and exposure time) and the Student-Newman-Keuls test at $p<0.05$ (Sokal and Rohlf, 1986).

\section{Results}

\section{Specificity of probes}

The specificity of the 2 ribosomal DNA probes, XX1.8 and pEE 6.7 is demonstrated in the series of light microscope autoradiographs in Figure 1. Since the external transcribed spacer region of the ribosomal gene is transcribed, processed, and degraded with the nucleolus (Hadjiolov, 1985), the pattern of labeling with the XX1.8 rDNA probe should be restricted to the nucleolus. Such a grain distribution, as well as low glia labeling and negligible background levels, were characteristically observed and are illustrated in Figure $1, A-C$. In Figure $1 A$, several neurons from the ventrolateral portion of the VL-VMN are shown. In each neuron, a cluster of centrally located grains lying over the nucleolus are present. No labeling over the cytoplasm is found and, in addition, background levels are low. In Figure $1 B$, a dense cluster of grains, lying in a central location within the nucleus of a VL-VMN neuron and obscuring the nucleolus, can be seen. In very lightly labeled cells (Fig. 1C), the outline of the nucleolus underneath the few grains confirmed the nucleolus-specific labeling with the XX1.8 rDNA probe. In Figure 


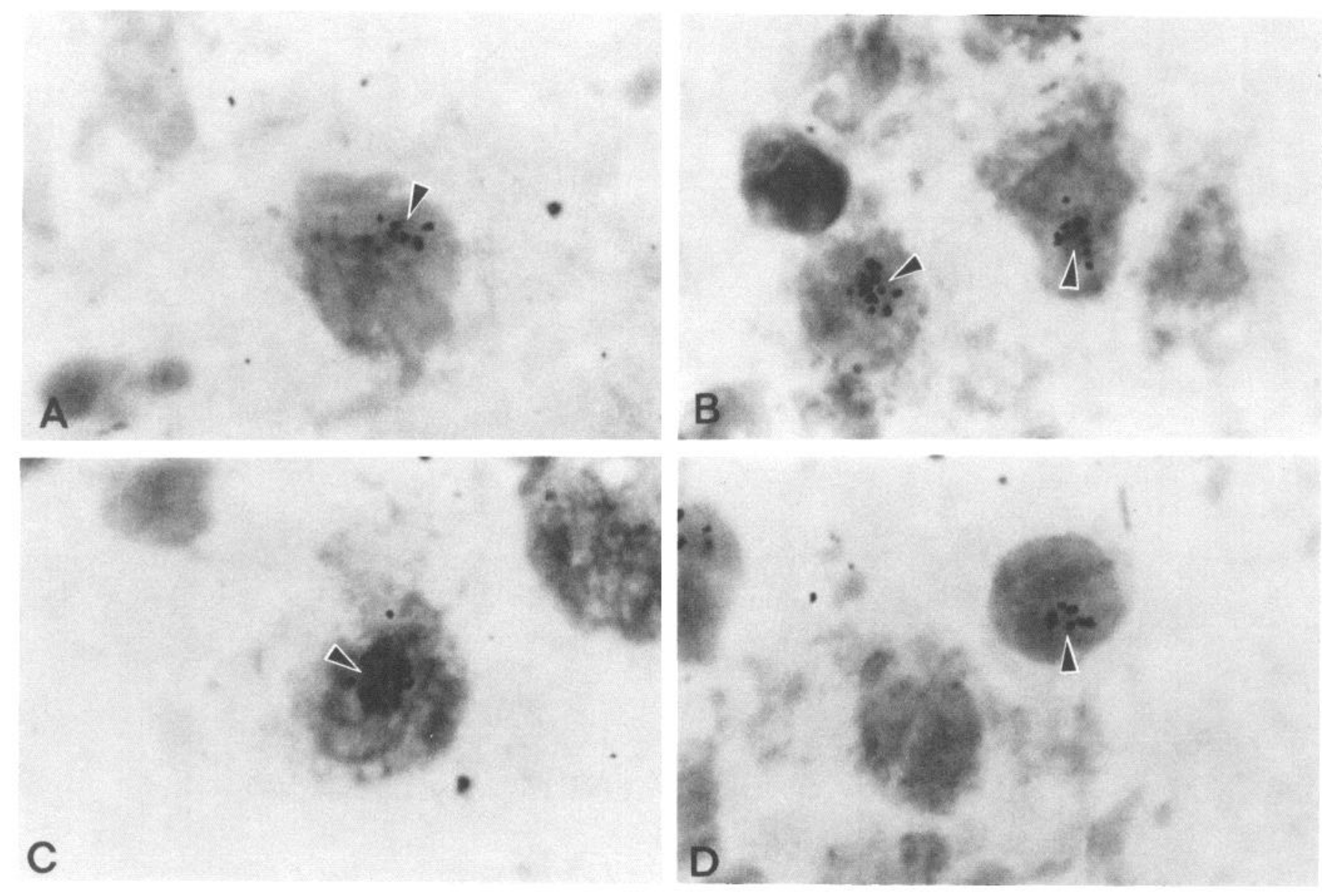

Figure 2. Autoradiographs of neurons from the ventrolateral portion of the ventromedial hypothalamus (VL-VMN) following hybridization with the precursor rDNA probe (XX1.8). In $A$, a VL-VMN neuron from an OVX rat is illustrated. Low levels of labeling can be seen over the nucleolus (arrowhead). In $B$, several VL-VMN neurons from OVX rats exposed to estradiol for $30 \mathrm{~min}$ are illustrated. Increased nucleolar labeling, relative to the controls illustrated in $A$, can be seen. In $C$, a VL-VMN neuron from OVX rats exposed to estradiol for $2 \mathrm{hr}$ is illustrated. Continued increase in the levels of precursor rRNA, as indicated by heavy labeling over the nucleolus, is apparent. In $D$, a VL-VMN neuron from OVX rats exposed to a second $2 \mathrm{hr}$ pulse of estradiol, $7 \mathrm{hr}$ after the first $2 \mathrm{hr}$ pulse of estradiol, is illustrated. Nucleolar labeling at the level of the control VL-VMN neurons is present.

$1 D$, the pattern of labeling with the pEE $6.7 \mathrm{rDNA}$ probe is shown. Since the pEE $6.7 \mathrm{rDNA}$ probe contains sequences found in mature ribosomes, the distribution of grains should be found throughout the cell. Such a grain distribution was consistently observed, as illustrated by the labeled VL-VMN neuron in Figure $1 D$. Furthermore, the pattern of hybridization with the $\mathrm{pEE}$ 6.7 rDNA probe included low levels of glial cell labeling and negligible background as previously described (McCabe et al., 1986; Jones et al., 1986).

\section{$E_{2}$ effects in the $V L-V M N$}

The effects of $E_{2}$ on the levels of precursor rRNA, reflected by the number of grains per neuron following hybridization with the XX1.8 rDNA probe, are illustrated by example in Figure 2 and quantitatively in Figure 3 . In Figure $2 A$, a VL-VMN neuron from an OVX rat, with relatively few grains overlying the nucleolus, is shown. In Figure 2, $B-D$, VL-VMN neurons from animals exposed to $E_{2}$ for $30 \mathrm{~min}, 2 \mathrm{hr}$, or $2 \mathrm{hr}$ on $/ 7 \mathrm{hr}$ off $/ 2 \mathrm{hr}$ on, respectively, are illustrated. Increasing numbers of grains overlying the nucleolus were found in VL-VMN neurons from the $30 \mathrm{~min}$ and $2 \mathrm{hr}$ experimental groups (cf. Fig. 2, $A$ to $B$, and $C$ ), but no difference in the numbers of grains overlying the nucleolus existed betwen neurons from OVX animals (Fig. 2A) and animals exposed to a second $2 \mathrm{hr}$ pulse of $\mathrm{E}_{2} 7 \mathrm{hr}$ after the first $2 \mathrm{hr}$ pulse (Fig. 2D). These changes were verified quantitatively using a computerized image-analysis system and under blind conditions (Fig. 3). No significant differences in the number of grains/neuron following hybridization with the XX1.8 rDNA probe were found in VL-VMN neurons after $15 \mathrm{~min}$ of $E_{2}$, whereas after $30 \mathrm{~min}$ and $2 \mathrm{hr}$ of $\mathrm{E}_{2}$ exposure, there were approximately 30 and $100 \%$ increases, respectively, in the number of grains/VL-VMN neuron relative to the control $[F(1,16)$ $=7.04, p<0.05]$. In the discontinuous hormone replacement paradigm, however, there was no difference between the numbers of grains/VL-VMN neuron in the OVX and $\mathrm{E}_{2}$-treated animals.

In contrast to the results obtained with the XX1.8 hybridization experiments, no differences in the levels of product rRNA, reflected by the number of grains/neurons following hybridization with the pEE 6.7 rDNA probe, were found in VL-VMN neurons at any of these early time points after hormone exposure (Figs. 3, 4).

In addition to the measurements of levels of rRNA, the effects of $E_{2}$ on the concentration of the specific rRNA sequences within 


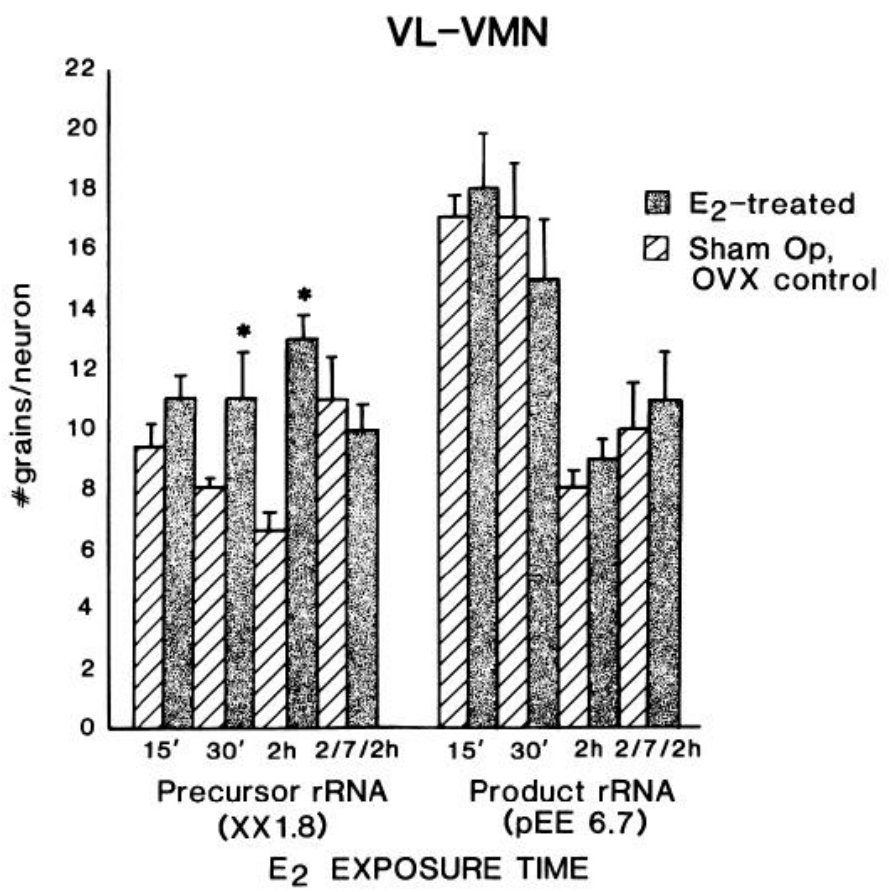

Figure 3. Effects of estradiol $\left(\mathrm{E}_{2}\right)$ on the number of grains per neuron within the ventrolateral portion of the ventromedial hypothalamus (VL$\mathrm{VMN}$ ). Using the XX1.8 rDNA probe to detect levels of precursor rRNA in each neuron, significant increases were noted after $30 \mathrm{~min}$ and $2 \mathrm{hr}$ of $\mathrm{E}_{2}(* ; p<0.05)$. Using the pEE 6.7 rDNA probe to detect levels of product (stable) rRNA, no significant differences were noted at any of the time points of $E_{2}$ exposure.

VL-VMN neurons were also determined. To accomplish this, measurements of somal area were first established using a computerized image-analysis system (Table 1). In our initial study of the effects of $E_{2}$ on product rRNA levels (Jones et al., 1986), the minimal hormone exposure time examined was $6 \mathrm{hr}$ of continuous $E_{2}$, which was sufficient to induce hypertrophy of VL-VMN neurons. In contrast, the shorter and discontinuous $\mathrm{E}_{2}$ exposure times used in the experiments in the present study did not result in any significant changes in the size of VL-VMN neurons (Table 1). Calculation of the concentration of precursor and product rRNA in individual VL-VMN neurons was accomplished by determining the grain density or number of grains per $\mu \mathrm{m}^{2}$ of somal area. As shown in Table 2, changes in the concentration paralleled the $\mathrm{E}_{2}$-induced changes or lack of changes in levels of precursor and product rRNA within individual VL-VMN neurons. No changes in the concentration of precursor rRNA were found after $15 \mathrm{~min}$ of $\mathrm{E}_{2}$ exposure, whereas significant increases in the concentration of precursor rRNA were found after $30 \mathrm{~min}$ and $2 \mathrm{hr}$ of $\mathrm{E}_{2}$ exposure, relative to the control $[F(1,16)=6.30, p<0.05]$. Exposure to a second $2 \mathrm{hr}$ pulse of $E_{2}, 7 \mathrm{hr}$ after the first $2 \mathrm{hr}$ pulse of $\mathrm{E}_{2}$, did not result in a secondary increase in the concentration of precursor rRNA. No changes in the concentration of product rRNA were found at any of the hormone exposure times examined (Table 2).

\section{$E_{2}$ effects in the ARC}

Accumulating evidence suggests that there is brain region specificity in the effects of steroid hormones. Our studies of the ultrastructural effects of $E_{2}$ on VL-VMN and ARC neurons, as

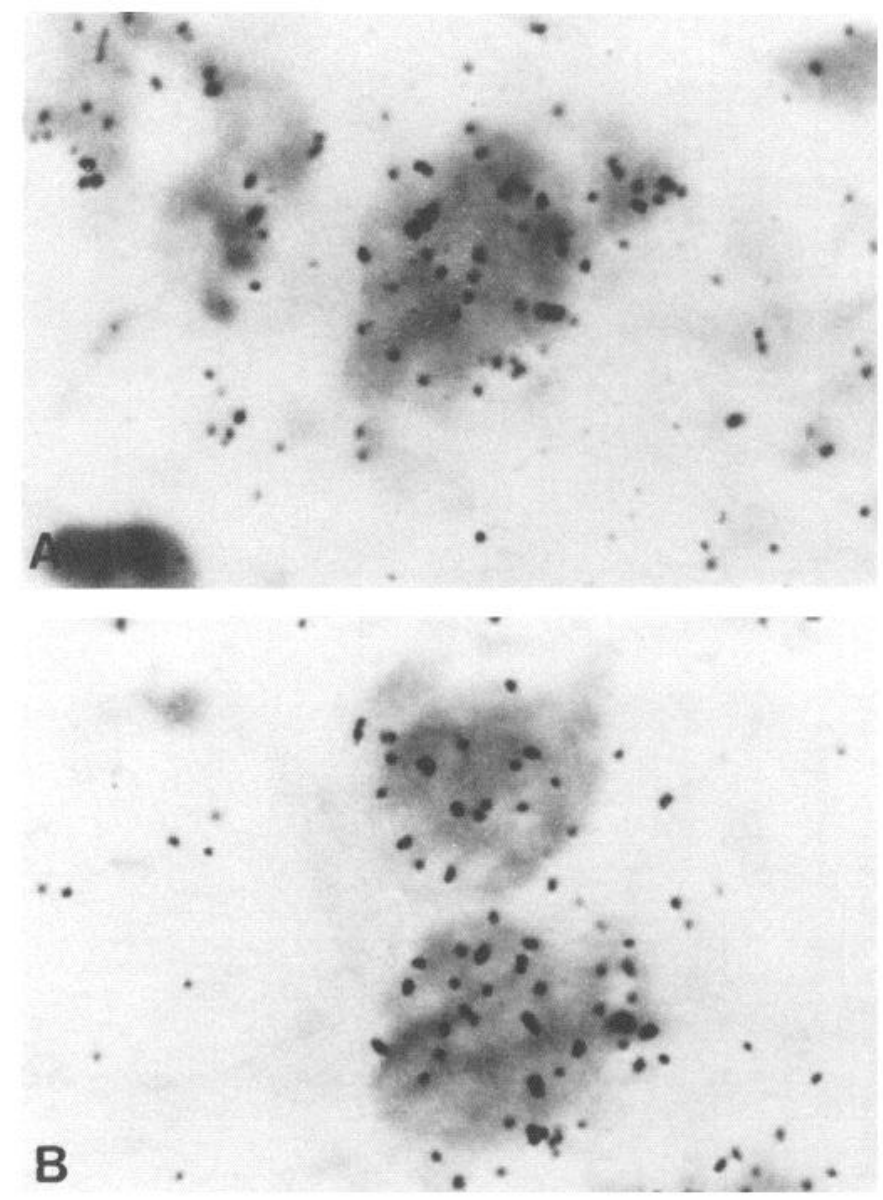

Figure 4. Autoradiographs of neurons from the ventrolateral portion of the ventromedial hypothalamus (VL-VMN) following hybridization with the product rDNA probes (pEE 6.7). No significant differences in the number or distribution of grains is observed between VL-VMN neurons from OVX control animals $(A)$ or OVX animals exposed for $2 \mathrm{hr}$ to estradiol $(B)$.

well as $\mathrm{E}_{2}$-induced changes in the ribosome synthesis (Jones et al., 1985, 1986, 1987a), show that the 2 types of neurons differ in their timing and magnitude of the response to the hormone. VL-VMN neurons undergo a more rapid and robust response to $\mathrm{E}_{2}$ than $\mathrm{ARC}$ neurons. Thus, $6 \mathrm{hr}$ of $\mathrm{E}_{2}$ administered to $\mathrm{OVX}$

Table 1. Measurements of somal area ${ }^{a}$

\begin{tabular}{llll} 
& \multicolumn{3}{l}{ Brain region $^{b}$} \\
\cline { 2 - 4 } V $_{2}$ exposure time & VL-VMN & ARC & DM-VMN \\
\hline $15 \mathrm{~min}$ & $140( \pm 7)$ & $63( \pm 3)$ & $110( \pm 12)$ \\
OVX control & $144( \pm 2)$ & $64( \pm 2)$ & $122( \pm 6)$ \\
$30 \mathrm{~min}$ & $123( \pm 11)$ & $64( \pm 6)$ & $114( \pm 9)$ \\
OVX control & $144( \pm 7)$ & $66( \pm 2)$ & $112( \pm 5)$ \\
$2 \mathrm{hr}$ & $129( \pm 6)$ & $62( \pm 2)$ & $109( \pm 8)$ \\
OVX control & $120( \pm 5)$ & $66( \pm 1)$ & $111( \pm 10)$ \\
$2 / 7 / 2 \mathrm{hr}$ & $126( \pm 8)$ & $60( \pm 3)$ & $109( \pm 13)$ \\
OVX control & $116( \pm 2)$ & $67( \pm 3)$ & $117( \pm 16)$ \\
\hline
\end{tabular}

${ }^{a}$ Somal area measurements are defined in $\mu \mathrm{m}^{2}$.

'VL-VMN, ventrolateral portion of the ventromedial nucleus of the hypothalamus; ARC, arcuate nucleus of the hypothalamus; DM-VMN, dorsomedial portion of the ventromedial nucleus of the hypothalamus. 


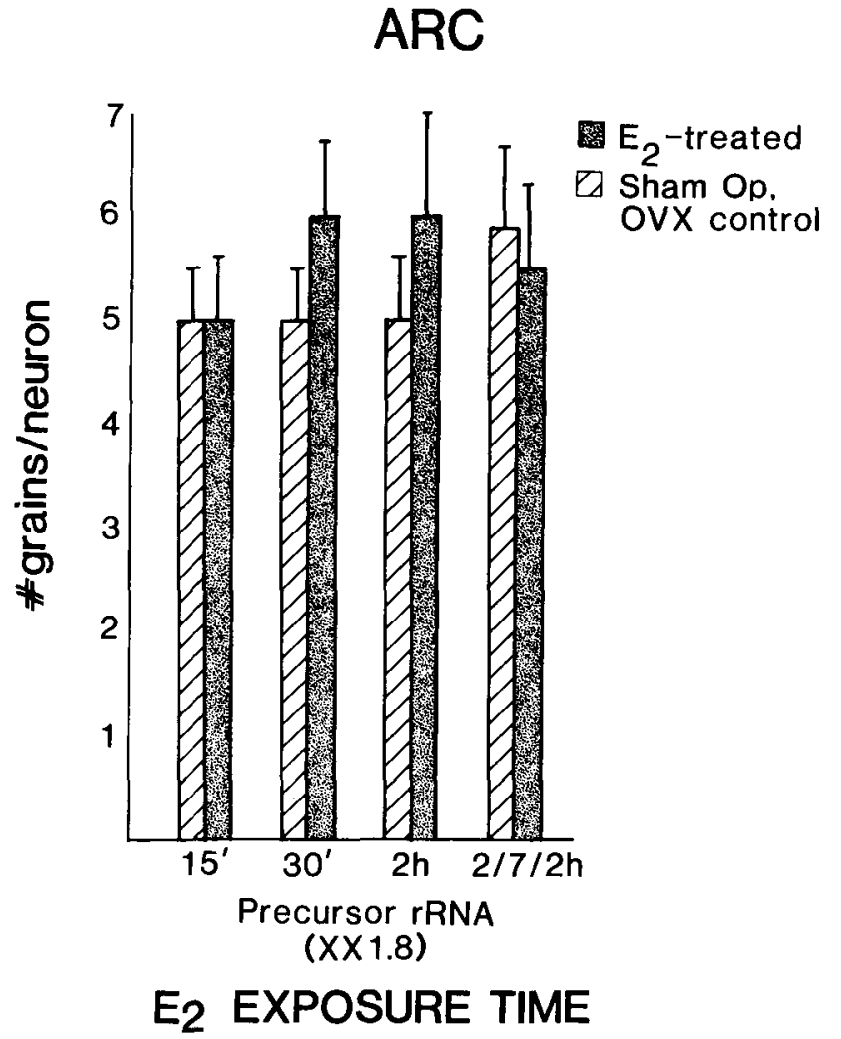

Figure 5. Effects of estradiol $\left(\mathrm{E}_{2}\right)$ on the number of grains within the arcuate nucleus of the hypothalamus (ARC). Using the XX1.8 rDNA probe to detect levels of precursor rRNA, no significant differences were noted at any of the time points of $E_{2}$ exposure. rats results in a $70 \%$ increase in product rRNA levels in VLVMN neurons but only a $30 \%$ increase in ARC neurons. Furthermore, $2 \mathrm{hr}$ of $\mathrm{E}_{2}$ administered to $\mathrm{OVX}$ rats results in significant morphological changes in VL-VMN neurons that are absent in similarly treated ARC neurons. To further characterize this brian region specificity, we also examined the effects of short-term and discontinuous exposure of $E_{2}$ in ARC neurons. The results corroborate the general trend of regional specificity of hormone action. In the ARC, no changes in levels of precursor rRNA, as determined by quantification of number of grains/ ARC neuron following hybridization with the XX1.8 rDNA probe, were found at any of the time points of $E_{2}$ exposure (Fig. 5). This lack of effect of $E_{2}$ on ARC neurons was reflected in the lack of changes in ARC somal area (Table 1) and concentration of precursor rRNA (Table 2) as well. Since no differences in levels of precursor rRNA were qualitatively observed, we did not quantify product rRNA in ARC neurons.

\section{Internal control: dorsomedial portion of the ventromedial nucleus (DM-VMN)}

The distribution of $\mathrm{E}_{2}$-concentrating neurons within the ventromedial nucleus of the hypothalamus is not homogeneous. Approximately $40 \%$ of the neurons within the VL-VMN concentrate $E_{2}$, whereas very few, if any, neurons within the DMVMN concentrate $E_{2}$. Since the DM-VMN is present in the same sections as the VL-VMN, we have routinely used the DM-VMN as an internal control, and, in this study, we examined the effects of $E_{2}$ on levels of precursor and product rRNA (Fig. 6), concentration of precursor and product rRNA (Table 1), and somal area (Table 2). No changes in any of the parameters quantified were found in the DM-VMN after any of the $E_{2}$ exposure times.

\begin{tabular}{|c|c|c|c|c|c|c|c|}
\hline \multirow{2}{*}{$\begin{array}{l}\text { Brain } \\
\text { region }\end{array}$} & \multirow[b]{2}{*}{ rRNA species } & \multicolumn{6}{|c|}{$\mathrm{E}_{2}$ exposure time } \\
\hline & & $15 \mathrm{~min}$ & $30 \mathrm{~min}$ & $2 \mathrm{hr}$ & & $2 / 7$ & $2 \mathrm{hr}$ \\
\hline \multirow[t]{6}{*}{ VL-VMN } & Precursor rRNA & & & & & & \\
\hline & OVX control & $6 \quad( \pm 0.1)$ & $6 \quad( \pm 0.6)$ & 5 & $( \pm 0.6)$ & 9 & $( \pm 1.0)$ \\
\hline & $\mathrm{E}_{2}$-treated & $7 \quad( \pm 0.6)$ & $9 \quad( \pm 1.0)^{d}$ & 10 & $( \pm 0.5)^{d}$ & 7 & $( \pm 1.0)$ \\
\hline & Product rRNA & & & & & & \\
\hline & OVX control & $11.7( \pm 0.8)$ & $15( \pm 1.6)$ & 7 & $( \pm 0.7)$ & 9 & $( \pm 0.9)$ \\
\hline & $\mathbf{E}_{2}$-treated & $11.4( \pm 1.0)$ & $12( \pm 1.0)$ & 6 & $( \pm 0.8)$ & 9 & $( \pm 0.9)$ \\
\hline \multirow[t]{3}{*}{ ARC } & Precursor rRNA & & & & & & \\
\hline & OVX control & $8 \quad( \pm 0.7)$ & $9 \quad( \pm 0.7)$ & 8 & $( \pm 1)$ & 8 & $( \pm 0.8)$ \\
\hline & $\mathrm{E}_{2}$-treated & $9 \quad( \pm 0.8)$ & $9 \quad( \pm 0.8)$ & 8 & $( \pm 0.6)$ & 10 & $( \pm 1.1)$ \\
\hline \multirow[t]{6}{*}{ DM-VMN } & Precursor rRNA & & & & & & \\
\hline & OVX control & $5.7( \pm 0.1)$ & $5.4( \pm 0.5)$ & & $( \pm 0.7)$ & & $( \pm 0.6)$ \\
\hline & $\mathrm{E}_{2}$-treated & $6.4( \pm 0.5)$ & $6.1( \pm 0.6)$ & & $( \pm 0.5)$ & & $( \pm 0.3)$ \\
\hline & Product rRNA & & & & & & \\
\hline & OVX control & $11.4( \pm 0.2)$ & $13.3( \pm 1)$ & 12.8 & $( \pm 1)$ & 10. & $( \pm 2)$ \\
\hline & $\mathrm{E}_{2}$-treated & $11.9( \pm 0.9)$ & $12.2( \pm 1)$ & 13.5 & $( \pm 0.9)$ & 11. & $( \pm 2)$ \\
\hline
\end{tabular}

\footnotetext{
Relative concentration of rRNA is defined as number grains $/ \mu \mathrm{m}^{2}$ somal area.

"Precursor rRNA was probed with a $1.8 \mathrm{~kb}$ DNA sequence excised with Xhol from the external transcribed region (XX1.8) at the beginning of the ribosomal gene. Product rRNA was probed with a $6.7 \mathrm{~kb}$ DNA sequence (pEE 6.7) that included portions of $18 \mathrm{~S}, 5.8 \mathrm{~S}$, and $28 \mathrm{~S}$ rDNA.

"VL-VMN, ventrolateral portion of the ventromedial nucleus of the hypothalamus (VMN); ARC, arcuate nucleus of the hypothalamus; DM-VMN, dorsomedial portion of the VMN.

Significant at $p<0.05$.
} 


\section{DM -VMN}



$E_{2}$-treated

Sham-operated, ovX control
Figure 6. Effects of estradiol $\left(\mathrm{E}_{2}\right)$ on the number of grains within the dorsomedial portion of the ventromedial nucleus (DM-VMN), a brain region with few $E_{2}$-concentrating neurons. No changes in the levels of precursor or product (stable) rRNA, as detected with the XX1.8 rDNA and pEE6.7 probe, respectively, were noted in this internal control region.

\section{Discussion}

In many non-neural steroid-sensitive tissues, hormonal regulation of the polymerase I system has been shown to be a major aspect of the mechanism by which steroids alter cellular functions. Until recently, no effects of steroids on nucleolar gene activity within neural target tissue had been identified. Our first experiments established that $\mathrm{E}_{2}$ exposure results in accumulation of large amounts of stable rRNA in hypothalamic neurons within $6 \mathrm{hr}$ (Jones et al., 1986). In the current study, tandem in situ hybridization experiments with precursor and product ribosomal DNA probes were employed to examine the effects of $\mathrm{E}_{2}$ on the production of ribosomes in individual neurons. This approach provided a novel means of quantifying the regulation of ribosome formation by estradiol at the level of the individual neurons.

$E_{2}$-induced changes in precursor rRNA levels were assayed by the use of tritium-labeled rDNA sequences complementary to the ETS. This ETS region is located upstream from the $18 \mathrm{~S}$, $5.8 \mathrm{~S}$, and $28 \mathrm{~S}$ rDNA regions, and is initially transcribed into a $45 \mathrm{~S}$ precursor molecule which is processed in the nucleolus to the stable rRNA species with the concomitant degradation of the ETS sequence (Chikaraishi et al., 1983). The ETS region has a short half-life and does not accumulate within cells (SollnerWebb and Tower, 1986). Thus, the rapid $E_{2}$-induced increases in the levels of precursor rRNA observed in the present study most likely represent direct effects of $E_{2}$ on the transcription of rRNA genes, as opposed to changes in the stability of the ribosomal transcripts. The $6.7 \mathrm{~kb}$ probe used to assess stable rRNA levels contains the internal transcribed spacer region, which, like the ETS, is rapidly degraded, and all of the $5.8 \mathrm{~S}$

rDNA, the majority of the $28 \mathrm{~S}$ rDNA, and a small fragment of $18 \mathrm{~S}$ rDNA. Although all of these sequences are found in the precursor 45S rRNA, the concentration of the precursor molecules is so much less than that of the stable rRNAs that the probe functionally detects only stable rRNAs at short exposure times. The results of experiments, both in the present study and in our previous work (Jones et al., 1986), using tritium-labeled rDNA sequences complementary to product rRNA, argue towards an effect of $E_{2}$ on rRNA transcription. Precursor rRNA accumulates following short exposures to $E_{2}$, whereas there is no detectable change in the level of product rRNA levels. Extrapolation of the data from the present study and our previous work (Jones et al., 1986) suggests that the converse is true following longer exposures to $E_{2}$. Precursor rRNA levels have returned to control values, whereas product rRNA levels have risen dramatically. Since the rate with which stable rRN $\Lambda$ levels should vary in response to changes in transcription depends on the half-life of the stable rRNAs, the long turnover for rRNAs would predict a long lag between changes in precursor transcription and accumulation of stable rRNAs. If estrogen increased the stability of the ribosomal precursor transcripts, resulting in accumulation of precursor rRNA, precursor rRNA levels would be expected to be elevated after longer exposures to $E_{2}$. Given that this is not the case, it would appear that $E_{2}$ increases rRNA transcriptional initiation. Since most of the effects of steroid hormones result from changes in transcription rates, the effects we describe here would be consistent with this mode of hormone action. As reviewed by Sollner-Webb and Tower (1986), the general availability of RNA polymerase I in cells, as well as the correlation of chromatin-bound polymerase I molecules and the rate of rRNA synthesis, suggests that stim- 
ulus-induced alterations in the rate of rRNA production are mediated at the level of transcriptional initiation and not at the level of transcript stability or elongation rate.

Comparison of the data obtained in the present study with evidence from other systems concerning the effects of steroid hormones on rRNA synthesis also corroborates the hypothesis that $E_{2}$ increases rRNA transcription in VMN neurons. Whelly and collaborators (Whelly, 1985, 1986; Whelly and Barker, 1986) have extensively studied $E_{2}$ effects on nucleolar functioning in the uterus. The results of their studies indicate that $E_{2}$ has a rapid effect on rRNA synthesis, with increases in rRNA transcription occurring within $1 \mathrm{hr}$ of exposure to the hormone. One of the trends to emerge from our studies of $E_{2}$ action on VMN neurons in rats is the remarkable similarity in the response characteristics of VMN neurons and uterine cells to the hormone. This similarity has been demonstrated ultrastructurally (Meisel and Pfaff, 1985), as well as temporally (Jones, 1988), and appears specific to neurons within the VL-VMN. Other hormone-responsive neuronal populations do not show a utcrine-like response to steroid hormone administration. Within the current study, no rapid effects of $E_{2}$ on rRNA synthesis in ARC neurons were noted at the time points examined.

It is important to note here that some degree of significant differences was observed between control groups. We have observed such intergroup variations in previous light microscope autoradiographic studies (Jones et al., 1986), which is one of the reasons why matched control groups were included for each hormone exposure time. The experimental design utilized in this study was such that paired hormone-treated and non-hormonetreated brain sections were processed together throughout the study. Thus, the most valid and consistent measure of analysis was that adopted in this investigation, i.e., comparisons were made between animals treated identically, with the exception of hormone exposure. When examining Figure 3, it can be seen that the values for the $2 \mathrm{hr}$ and $2 / 7 / 2 \mathrm{hr}$ groups hybridized with product rDNA appear lower than those in the 15 and $30 \mathrm{~min}$ groups. This is due, in part, to the fact that these groups comprised different hybridization runs. Also in Figure 3, there is a significant level of intergroup variability between the $2 \mathrm{hr}$ and $2 / 7 / 2$ hr control VMN groups (Fig. 3, precursor rRNA). It is possible that $\mathrm{E}_{2}$ could simply be preventing a decrease in precursor rRNA brought about by the stress of anesthesia blank capsule insertion. However, this is unlikely, given that a large decrease in the control values following the second $2 \mathrm{hr}$ shaminsertion did not occur. An alternate explanation could be inherent metabolic differences, expressed in terms of basal ribosomal RNA levels, due to diurnal rhythmic modifications taking place at the cellular level. Pebusque and Seite $(1980,1981)$ found evidence of a circadian rhythm in the nucleolus of the rat sympathetic neurons that included nucleolar hypertrophy during the latter stages of the dark period. In a morphological study of the nuclear and nucleolar effects of $E_{2}$ on VMN neurons, we found very little differences between nuclear shape, nuclear area, or nuclear perimeter between control $2 \mathrm{hr}$ and $2 / 7 / 2 \mathrm{hr}$ animals (Jones et al., 1985). However, there were significant differences between the nucleolar area of animals in these 2 groups, with the nucleolar area being larger in the $2 / 7 / 2 \mathrm{hr}$ control group than in the $2 \mathrm{hr}$ control group. This supports the hypothesis of diurnal variations in nucleolar activity contributing to differences between control groups observed in this study.

Several lines of evidence indicate that $E_{2}$ acts very early and possibly in a discontinuous manner within the VMN to effect behavioral changes in the OVX rat (Parsons et al., 1982a, b; Jones et al., 1988). Nucleolar hypertrophy in VMN neurons occurs within the first $2 \mathrm{hr}$ exposure to $\mathrm{E}_{2}$ in OVX rats, recedes after removal of the $E_{2}$ implant, and does not recur during the second exposure to the hormone (Jones et al., 1985). Since nucleolar hypertrophy has been firmly established to be a morphological index of increased rRNA transcription (Busch and Smetana, 1970), our results here indicating that precursor rRNA levels rise after the first, but not the second, $2 \mathrm{hr}$ pulse of $\mathrm{E}_{2}$ in VMN neurons correlate well temporally with the morphological data and support our conclusion that $E_{2}$ acts at the level of rRNA transcription.

Estrogenic activation of the ribosomal genes in VL-VMN neurons within $30 \mathrm{~min}$ is the earliest event in the mechanism of hormonal action on the brain elucidated to date. The lack of any changes in VL-VMN neuronal size after very short exposures to $E_{2}$, in view of the known growth-related effect of $E_{2}$ on these neurons after longer exposures (Jones et al., 1986), as well as the widespread effect of $E_{2}$ on protein synthesis in the VMN (Scouten et al., 1985; Jones et al., 1987, 1988), suggest a primary effect of steroid hormones on the nucleolus in VMN neurons. The nucleolar response may then mediate some of the other neurotrophic actions of these hormones. The determination of such an early effect of gonadal steroids on target neurons establishes an important temporal benchmark for future studies directed at the identification of transcriptional factors or other early immediate genes that may be activated in response to steroid administration.

\section{References}

Barfield RP, Chen J (1977) Activation of estrous behavior in ovariectomized rats by intracerebral implants of estradiol benzoate. Endocrinology 101:1716-1725

Bolender RP (1979) Surface area ratios. I. A stereological method for estimating average cell changes in membrane surface areas. Anat Rec 194:511-522.

Breedlove SM (1986) Cellular analyses of hormone influence on motoneuronal development and function. J Neurobiol 17:157-176.

Busch H, Smetana K (1970) The nucleolus. New York: Academic Press.

Chikaraishi DM, Buchanan L, Danna KJ, Harrington CA (1983) Genomic organization of rat rDNA. Nucleic Acids Res 11:6437-6452.

Cochran WG (1953) Sampling techniques. New York: Wiley.

Cohen RS, Pfaff DW (1981) Ultrastructure of neurons on the ventromedial nucleus of the hypothalamus in ovariectomized rats with or without estrogen treatment. Cell Tissue Res 217:451-470.

Davis PG, Krieger MS, Barfield RJ, McEwen BS, Pfaff DW (1982) The site of action of intrahypothalamic estrogen implants in feminine sexual behavior: an autoradiographic analysis. Endocrinology 111: 1581-1586.

DeVoogd TJ (1986) Steroid interactions with structure and function of avian song control regions. J Neurobiol 17:177-201.

Hadjiolov AA (1985) The nucleolus and ribosome biogenesis. New York: Springer.

Jensen EV, Greene GL, Closs LE, DeSombre ER, Nadji M (1982) Receptors reconsidered: a 20-year perspective. Rec Prog Horm Res $38: 1-40$.

Jones KJ (1988) Steroid hormones and neurotrophism: relationship to nerve injury. Metab Brain Dis 3:1-17.

Jones KJ, Pfaff DW, McEwen BS (1985) Early estrogen-induced nuclear changes in rat hypothalamic ventromedial neurons: an ultrastructural and morphometric analysis. J Comp Neurol 239:255-266.

Jones K, Chikaraishi D, Harrington C, McEwen BS, Pfaff DW (1986) In situ hybridization detection of estradiol-induced changes in ribosomal RNA levels in rat brain. Mol Brain Res 1:145-152.

Jones KJ, McEwen BS, Pfaff DW (1987) Quantitative assessment of the synergistic and independent effects of estradiol and progesterone on ventromedial hypothalamic and preoptic area proteins in female rat brain. Metab Brain Dis 2:271-281 
Jones KJ, McEwen BS, Pfaff DW (1988) Quantitative assessment of early and discontinuous estradiol-induced effects on ventromedial hypothalamic and preoptic area proteins in female rat brain. Neuroendocrinology (in press).

Lewis EJ, Tank AW, Weiner N, Chikaraishi DM (1983) Regulation of tyrosine hydroxylase mRNA by glucocorticoid and cyclic AMP in a rat pheochromocytoma cell line. J Biol Chem 258:14632-14637.

Mathews MD, Fdwards DA (1977) The ventromedial nucleus of the hypothalamus and the hormonal arousal of sexual behaviors in the female rat. Horm Behav 4:40-51.

McCabe JT, Morrell JI, Pfaff DW (1986) In situ hybridization technique to localize rRNA and mRNA in mammalian neurons. J Histochem Cytochem 34:45-50.

Meisel RL, Pfaff DW (1985a) Brain region specificity in estradiol effects on neuronal ultrastructure in rats. Mol Cell Endocrinol 40: $159-166$.

Meisel RL, Pfaff DW (1985b) Specificity and neural sites of action of anisomycin in the reduction or facilitation of female sexual behavior in rats. Horm Behav 19:237-251.

Mobbs CV, Harlan RE, Burrous MR, Pfaff DW (1988) An estradiolinduced protein synthesized in the ventral medial hypothalamus and transported to the midbrain central gray. J Neurosci 8:113-118.

Morrell JI, Pfaff DW (1983) Immunocytochemistry of steroid hormone receiving cells in the CNS. Methods Enzymol 103:639-662.

Moss RL, Foreman MM (1976) Potentiation of lordosis behavior by intrahypothalamic infusion of synthetic luteinizing hormone-releasing hormone. Neuroendocrinology 20:176-181.

Parsons B, McEwen BS, Pfaff DW (1982a) A discontinuous schedule of estradiol treatment is sufficient to activate progesterone-facilitated feminine sexual behavior and to increase receptors for progestins in the hypothalamus of the rat. Endocrinology 110:613-619.

Parsons B, Rainbow TC, Pfaff DW, McEwen BS (1982b) Hypothalamic protein synthesis essential for the activation of the lordosis reflex in the female rat. Endocrinology 110:620-625.
Pebusque M-J, Seite R (1980) Circadian change of fibrillar centers in nucleolus of sympathetic neurons: an ultrastructural and stereological analysis. Biol Cell 37:219-222.

Pebusque MJ, Seite R (1981) Evidence of a circadian rhythm in nucleolar components of rat superior cervical ganglion neurons with particular reference to the fibrillar centers: an ultrastructural and stereological analysis. J Ultrastruct Res 77:83-92.

Pfaff DW (1980) Estrogens and brain function. New York: Springer.

Pfaff DW, Keiner M (1973) Atlas of estradiol-concentrating cells in the central nervous system of the female rat. J Comp Neurol 151 : 121-158.

Scouten CW, Heydorn WE, Creed GJ, Malsbury CW, Jacobowitz DM (1985) Proteins regulated by gonadal steroids in the medial preoptic and ventromedial hypothalamic nuclei of male and female rats. Neuroendocrinology 41:237-245.

Sokal RR, Rohlf RJ (1981) Biometry: the principles and practice of statistics in biological research. San Francisco: WA Freeman.

Sollner-Webb B, Tower J (1986) Transcription of cloned eukaryotic ribosomal RNA genes. Annu Rev Biochem 55:801-830.

Vic P, Garcia M, Humeau C, Rochefort H (1980) Early effect of estrogen on chromatin ultrastructure in endometrial nuclei. Mol Cell Endocrinol 19:79-92.

Weibel ER, Bolender RP (1973) Stereological techniques for electron microscopic morphometry. In: Principles and techniques of electron microscopy, Vol 3 (Hayat MA, ed), pp 237-296. New York: Van Nostrand Reinhold.

Whelly SM (1985) Regulation of uterine nucleolar RNA synthesis by estrogens. Biol of Rep 33:1-10.

Whelly SM, Barker KL (1985) Inhibition of the aminoacylation of selected tRNA molecules by an estrogen-related factor on uterine ribosomes. Eur J Biochem 146:245-253. 